\title{
Downlink Capacity Analysis of Collaborative Crossed Timeslots in CDMA TDD Systems
}

\author{
Ki Won Sung, Chae Y. Lee, Kwang Hyuk Yim and Agnes Gravrand* \\ Dept. of Industrial Engineering, KAIST, 373-1 Kusung Dong, Taejon, Korea \\ Tel: +82-42-869-2916, FAX: +82-42-869-3110, Email: chae@kaist.ac.kr \\ *Dept. of Telecommunications, Services and Uses, INSA, Lyon, France
}

\begin{abstract}
Traffic asymmetry between uplink and downlink is expected to be a remarkable 3G characteristic in cellular mobile multimedia communications. CDMA system with TDD is a good solution to this traffic asymmetry. However, the level of traffic asymmetry may be significantly different from a cell to another cell. To tackle this problem and to support the traffic hot spot, crossed slots are examined. The use of crossed slots is restricted within a certain range of a cell by investigating the inter-cell interference and the maximum planned load factor. We examine the radius of inner zone and discuss the capacity of downlink crossed slots for various neighbor cell environments. Computational results show that the capacity increase in the target cell is outstanding by reducing the service range of neighbor cells. When all six neighbors reduce their service range by $20 \%$, the capacity at target cell crossed slot is increased by 35\%. Monte Carlo simulation is performed with large scale fading to verify the numerical analysis.
\end{abstract}

\section{Keywords}

CDMA-TDD, traffic asymmetry, crossed slot, inner zone, inter-cell interference 


\section{Introduction}

Many multimedia services of next generation mobile telecommunication systems have an asymmetric traffic between uplink (UL) and downlink (DL). In spite of the high capacity offered by the symmetric FDD mode of UMTS, the DL bandwidth may be saturated while the UL bandwidth is not fully used. To alleviate this problem, the TDD mode is a good solution with the CDMA technique [1].

In CDMA-TDD system, downlink and uplink are separated by different timeslots on the same frequency. TDD system is capable of managing traffic asymmetry by assigning different number of timeslots to DL and UL according to their traffic loads. TDD system thus has flexibility to control the UL and DL traffic load. However, when the rate of asymmetry is different in each cell, it leads to additional inter-cell interference problem. If two adjacent cells independently allocate timeslots according to their traffic asymmetries, same timeslots will be used for DL in one cell, and for UL in the other cell. These timeslots are called crossed slots [2]. Figure 1 illustrates the crossed slot interference problem. In the figure, timeslots are used for DL in cell 1 and UL in cell 2.

Base station (BS)1 in DL interferes with BS2 in UL, which causes BS-to-BS interference. At the same time, mobile station (MS)2 becomes an interference source to MS1 in the

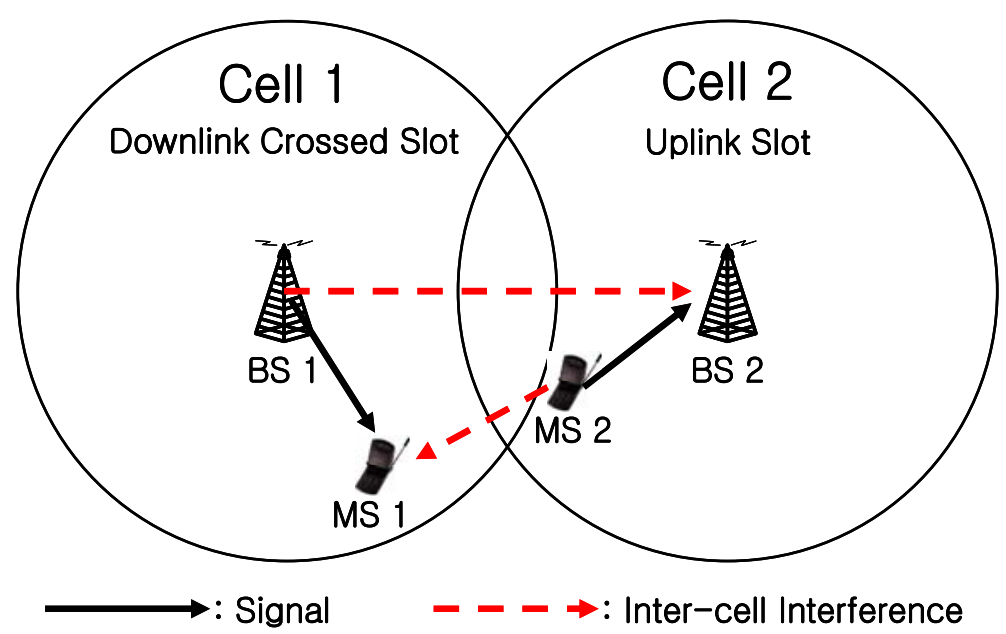

Figure 1. Crossed slot interference problem 
neighbor cell, which is called mobile-to-mobile interference. It is reported that BS-to-BS interference substantially decreases the system capacity [3]. Mobile-to-mobile interference may also result in degradation of performance when interfering and interfered mobiles are close to the cell boundary [4]. Jeon and Jeong [2] and Song Ni and Lajos Hanzo [5] showed that careful timeslot allocation outperforms the non-crossed slot allocation in terms of the maximum capacity and forced termination probability.

Several papers have been released to solve the crossed slot interference problem. Beamforming [6-8] enables a BS to focus its transmitted signal energy on the served MSs. Thus, it decreases the inter-cell interference at adjacent BSs. However, this desirable goal may only be achieved, if there are no MSs that use the crossed slot in the line between the serving BS and the neighboring BS.

Zone division scheme $[2,9]$ has the advantage of simplicity. It simply divides each cell into inner zone and outer zone and assigns crossed slots to mobiles in inner zone with reduced power. The performance of the zone division scheme is studied in [2, 9-10]

It should be noted that the main purpose of the zone division is to reduce BS-to-BS and mobile-to-mobile inter-cell interference. Thus, the size of inner zone is one of the most important parameters to be determined. We in this paper investigate the maximum size of inner zone for the crossed slots when adjacent cells collaboratively control the transmission power. The traffic amount that can be covered by the crossed slots is also examined.

The remainder of this paper is organized as follows. In Section 2, the system model and timeslot allocations are explained. In Section 3, capacity analysis of DL crossed slots is analyzed mathematically. We employ four scenarios and figure out capacity of crossed slots in downlink. In Section 4, numerical results are presented. Simulation results are also presented to verify the results of analysis. Finally, conclusion is provided in Section 5. 


\section{System model and Distributed Timeslot Allocation}

To investigate the size of inner zone that can employ downlink crossed slots, we adopt TDD mode in UMTS Terrestrial Radio Access system, which is TD-CDMA technology [13]. A 10 ms frame is divided into 15 timeslots. A timeslot can be assigned for either downlink or uplink. We assume that same modulation scheme is used in uplink and downlink. Therefore, data rate of a timeslot per code is fixed to 8 Kbps by considering spreading factor 16 with $1 / 2$ rate channel coding in both links. We assume single frequency allocation.

By assuming distributed timeslot allocation of each radio network controller (RNC), the RNC collects DL and UL traffic loads in every cell. Then, it divides the 15 timeslots into DL and UL according to the average traffic loads. This is the RNC guideline of DL and UL timeslot allocation for its cells. This guideline is called nominal slot allocation. However, some cells may use more DL timeslots. If a cell uses timeslots at DL against the nominal slot allocation, we call these timeslots DL crossed slots.

To solve the interference problem in the implementation of crossed slots, a cell with crossed slots needs to regulate its interference to adjacent cells. To make DL crossed slots behave just like nominal timeslots in terms of interference we propose the following two conditions for the use of crossed slots.

Condition 1-DL: Inter-cell interference from a BS in cell of DL crossed slot should not exceed that from mobiles in a cell of nominal UL slot.

Condition 2-DL: The $E_{b} / N_{0}[14,16]$ in a cell of DL crossed slot should be higher than the minimum required DL $E_{b} / N_{0}$.

Under these conditions, we will investigate the maximum range of inner zone of a target cell and the capacity of crossed slots in different situations. We assume the distance between a 
mobile and a base station is known by using GPS [11, 12]. As shown in Figure 2, when cell B with nominal slots reduces its service range, cell A with DL crossed slots can increase its inner zone coverage and DL traffic. This situation is realistic when the traffic in cell B is sparse and mobiles out of service range can be covered by other UL timeslots.

In order to analyze the situation in multi-cell environment, we assume that a target cell with DL crossed slots is surrounded with six cells. We classify the surrounded six cells into the following three groups depending on the DL and UL traffics.

Group A: cells which use DL crossed slots that are equal to the target cell

Group B: cells which use nominal UL slots and reduce service range

Group C: cells which use nominal UL slots and don’t reduce service range

Cells in Group A have larger DL traffic than UL compared to the cells that use nominal UL slots. Cells in Group B have less UL traffic than cells in Group C enough to reduce their service range. In this study we examine the size of inner zone and capacity increase of crossed slots of a target cell when adjacent cells are in one of the above three groups. We also investigate cases when adjacent cells are combinations of Group A, B and C.

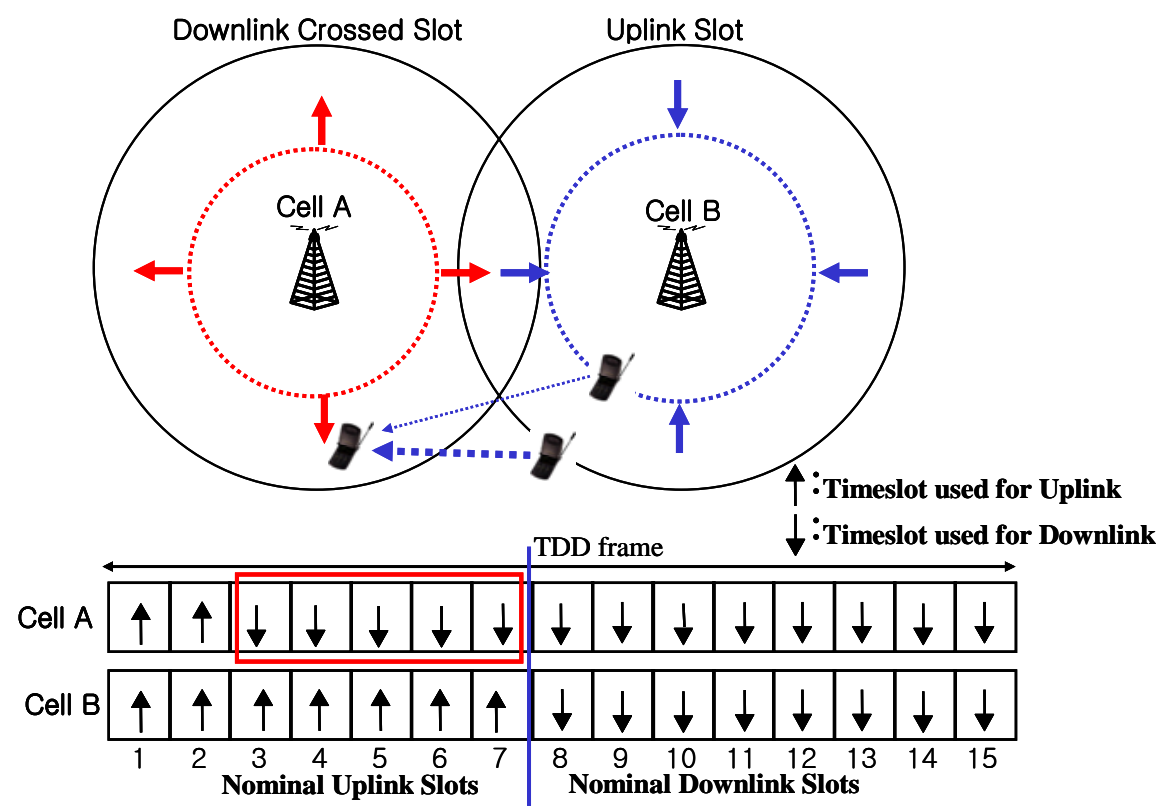

Figure 2. Inner zone of cell A can be enlarged when cell B reduces its range. 


\section{Capacity analysis of downlink crossed slots}

To simplify our analysis we assume circular cells with average path loss given by $\mathrm{kd}^{-v}$. Cells are overlapped in their boundary to allow for handover margin. Notations used throughout the paper are listed in Table 1 . In the notation superscripts $\mathrm{D}$ and $\mathrm{U}$ represent DL and UL respectively. The other superscripts A, B, C and T indicate Group A, Group B, Group C and the target cell respectively.

\subsection{Analysis between target cell and adjacent cells in Group C}

In this first scenario, the target cell employs DL crossed slots and all adjacent cells use nominal UL slots in its full range. To have a practical bound of the size of inner zone, we consider the worst case mobile in DL crossed slots. Thus, mobile $t$ in DL crossed slot is assumed to be located on the circle with radius $r$.

Table 1. Notations

\begin{tabular}{cc}
\hline Symbol & Definition \\
\hline$W$ & Spreading bandwidth \\
$S$ & Data rate of an RU \\
$v$ & Number of slots in a frame \\
$k$ & Path loss exponent \\
$\alpha$ & Orthogonality factor in DL \\
$L$ & Number of adjacent cells \\
$D$ & Radius of a cell \\
$r$ & Transmission power of a target BS for DL crossed slots \\
$P^{D, T}$ & Transmission power of a mobile in a cell (UL slot) when the distance from BS is $x$ \\
$P_{x}^{U}$ & Number of mobiles in a cell in a timeslot \\
\hline
\end{tabular}


To decide the base station power of the target cell that satisfies Condition 1-DL, let $P^{D, T}$ be the transmission power at target BS for DL crossed slot. Also, let $I^{D, T}$ be the interference power from target BS received at an adjacent BS in Group C, $I^{U, C}$ be the average inter-cell interference that a mobile generates and $n_{\max }^{U}$ be the number of mobiles that can be served in one UL timeslot. By Condition 1-DL, inter-cell interference from target BS has to be less than total inter-cell interference from MSs in another cell of Group C as in Figure 3. Therefore, the inequality $I^{D, T}=P^{D, T} k D_{B B}{ }^{-V} \leq n_{\max }^{U} I^{U, C}$ should be satisfied, where $D_{B B}$ is the distance between two BSs. Thus, the maximum transmission power at the target cell $P^{D, T}$ is determined by the following equation.

$$
P^{D, T}=\frac{n_{\max }^{u} I^{U, C}}{k D_{B B}{ }^{-v}}
$$

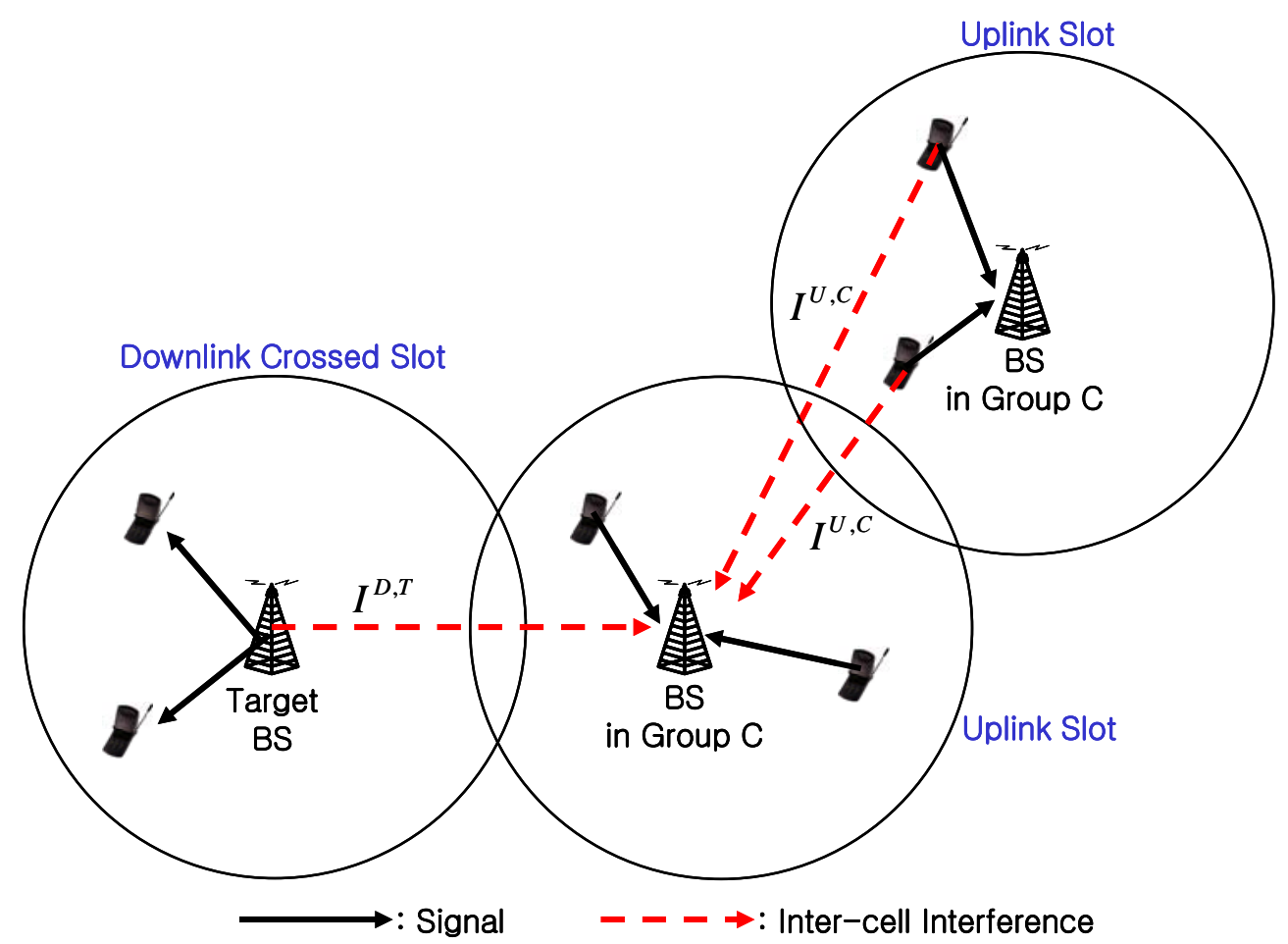

Figure 3. Inter-cell interference from downlink crossed slot and uplink slot 
Now, for Condition 2-DL, we need to compute mobile-to-mobile inter-cell interference from cells in Group C. We employ polar coordinate. We assume that the target BS is located at $(0,0)$ and the tagged mobile $t$ in the target cell is at $(r, 0)$. Mobile $t$ receives mobile-tomobile inter-cell interference from mobiles using nominal UL slots in adjacent cells in Group C. We assume mobiles in Group C are uniformly distributed. Let us consider adjacent cell $j$ whose BS is located at $\left(D_{B B}, \frac{2 \pi j}{L}\right)$. When we denote $D_{j t}(r)$ as the distance between mobile $t$ in the target cell and BS $j$, it is given by

$$
D_{j t}(r)=\sqrt{D_{B B}^{2}+r^{2}-2 D_{B B} r \cos \frac{2 \pi j}{L}}
$$

To obtain distance from mobile $t$ and an arbitrary mobile $m$ in cell $j$, we consider another coordinate where the position of BS $j$ is $(0,0)$ and that of mobile $t$ is $\left(D_{j t}(r), 0\right)$. Now, consider an arbitrary mobile $m$ in cell $j$ whose position is $(a, \theta)$. Then, the distance between mobile $t$ and $m, D_{m j t}(a, \theta, r)$, is given by

$$
D_{m j t}(a, \theta, r)=\sqrt{D_{j t}^{2}(r)+a^{2}-2 D_{j t}(r) a \cos \theta}
$$

Let $I_{m j t}^{U, C}(a, \theta, r)$ be the interference power that mobile $t$ in the target cell receives from mobile $m$ in adjacent cell $j$. From Equation (3), $I_{m j t}^{U, C}(a, \theta, r)$ is given by

$$
I_{m j t}^{U, C}(a, \theta, r)=k P_{a}^{U} D_{m j t}(a, \theta, r)^{-v} \text {, where } P_{a}^{U}=P_{D}^{U} \frac{D^{-v}}{a^{-v}}
$$

Thus, $\bar{I}_{\text {mjt }}^{U, C}(r)$, the expected value of $I_{m j t}^{U, C}(a, \theta, r)$, can be expressed as follows.

$$
\begin{aligned}
\bar{I}_{m j t}^{U, C}(r) & =\int_{0}^{D} \int_{0}^{2 \pi} I_{m j t}^{U, C}(a, \theta, r) \frac{a}{\pi D^{2}} d \theta d a \\
& =\frac{k P_{D}^{U} D^{-(v+2)}}{\pi} \int_{0}^{D} \int_{0}^{2 \pi} a^{v+1}\left(D_{j t}{ }^{2}(r)+a^{2}-2 D_{j t}(r) a \cos \theta\right)^{-v / 2} d \theta d a
\end{aligned}
$$

Since $n_{\max }^{U}$ mobiles simultaneously transmits signals in cell $j$, the total amount of interference from cell $j$ to mobile $t, I_{j}^{U, C}(r)$, is computed by 


$$
I_{j}^{U, C}(r)=n_{\max }^{u} \bar{I}_{m j t}^{U, C}(r)
$$

Let $\left(E_{b} / N_{0}\right)^{D, T}$ be the $E_{b} / N_{0}$ value of a mobile in DL crossed slots and $n_{\max }^{D}$ be the maximum number of mobiles in a DL slot. Then, we have

$$
\left(\frac{E_{b}}{N_{0}}\right)^{D, T}=\frac{W}{S R} \frac{k P^{D, T} r^{-v} / n_{\max }^{D}}{(1-\alpha) k P^{D, T} r^{-v}+\sum_{j=1}^{L} I_{j}^{U, C}(r)+P_{N}}
$$

By letting the above $E_{b} / N_{0}$ value at mobiles in the DL crossed slots be the same as the $E_{b} / N_{0}$ requirement $\gamma^{D}$ for DL service, the maximum allowable size of inner zone can be obtained. When the radius of the target cell is $0.65 \mathrm{Km}$, the maximum size of inner zone that meets $\gamma^{D}=7 \mathrm{~dB}$ becomes $r=0.38 \mathrm{Km}$ which corresponds to $58.8 \%$ of the cell radius.

\subsection{Analysis between target cell and adjacent cells in Group B}

In the second scenario, we analyze the target cell which is adjacent to cells in Group B. It should be noted that neighboring cells are belong to Group B only when their uplink traffic load is small enough to reduce their service range.

When the adjacent cells in Group B reduce their service range, they generate reduced intercell interference. Therefore, inner zone of the target cell is enlarged. As in Section 3.1 the base station power of the target cell is restricted by Equation (1). For Condition 2-DL, we assume the reduced transmission range of a cell in Group B is $D^{\prime}$ and mobiles are distributed within the range. Then, the average mobile-to-mobile interference in the second scenario is computed as

$$
\begin{aligned}
\bar{I}_{m j t}^{U, B}(r) & =\int_{0}^{D^{\prime}} \int_{0}^{2 \pi} I_{m j t}^{U, B}(a, \theta, r) \frac{a}{\pi D^{\prime 2}} d \theta d a \\
& =\frac{k P_{D}^{U} D^{-v}}{\pi D^{\prime 2}} \int_{0}^{D^{\prime}} \int_{0}^{2 \pi} a^{v+1}\left(D_{j t}{ }^{2}(r)+a^{2}-2 D_{j t}(r) a \cos \theta\right)^{-v / 2} d \theta d a
\end{aligned}
$$


By computing total amount of interference from cell $j$ to mobile $t$ as in Equation (6), the $E_{b} / N_{0}$ of a mobile in DL crossed slots is computed as

$$
\left(\frac{E_{b}}{N_{0}}\right)^{D, T}=\frac{W}{S R} \frac{k P^{D, T} r^{-v} / n_{\max }^{D}}{(1-\alpha) k P^{D, T} r^{-v}+\sum_{j=1}^{L} I_{j}^{U, B}(r)+P_{N}}
$$

From Equation (9) the maximum radius $r$ of the inner zone of the target cell is obtained when $\left(E_{b} / N_{0}\right)^{D, T}$ equals to the minimum requirement $\gamma^{D}$ in the DL crossed slot. In this scenario, due to the reduced transmit power of cells in Group B, inner zone of the target cell satisfying the Condition 2-DL is enlarged compared to the first scenario in Section 3.1. When all six adjacent cells reduce their service range to $50 \%$ of the cell radius, the inner zone is expanded to $72.8 \%$ of the cell radius.

\subsection{Analysis between target cell and adjacent cells in Group A}

In the third scenario, all adjacent cells employ DL crossed slots as the target cell. By Condition 1-DL, the target cell base station power is limited by $P^{D, A}=\frac{n_{\max }^{u} I^{U, C}}{k D_{B B}{ }^{-v}}$ from Equation (1). With the distance $D_{j t}(r)$ between base station $j$ in Group A and mobile $t$ in the target base station, the interference from the cells in Group A is computed as

$$
I_{j}^{D, A}(r)=k P^{D, A} D_{j t}^{-v}(r)
$$

Note that $P^{D, A}$ is regulated so that a BS of DL crossed slot has less inter-cell interference than that from a cell of Group C. In other words, $I_{j}^{D, A}$ is regulated not to exceed $I_{j}^{U, C}(r)$. Thus, the DL crossed slot of adjacent cells can be regarded as that of nominal UL slot in view of inter-cell interference. 


\subsection{Analysis between target cell and adjacent cells in Group A, B and C}

When adjacent cells are combination of Group A, B and C, inter-cell interference from adjacent cells depends on the location of mobile $t$ in the target cell. Thus, let the location of mobile $t$ in the target cell be $(r, \delta)$, then the distance $D_{j t}(r, \delta)$ between the base station in the adjacent cell $j$ and mobile $t$ becomes

$$
D_{j t}(r, \delta)=\sqrt{D_{B B}{ }^{2}+r^{2}-2 D_{B B} r \cos \left(\frac{2 \pi j}{L}-\delta\right)}
$$

Using Equation (11), average inter-cell interference can be computed by location the mobile at $(r, \delta)$. Thus, the average inter-cell interference is not dependent on the locations of cells in Group A, B and C. However, it is dependent on the number of cells in each group.

Let $\mathrm{b}$ and $\mathrm{c}$ represent the number of cells in Group B and C respectively. Then the $E_{b} / N_{0}$ of a mobile which uses the DL crossed slots is computed as

$$
\left(\frac{E_{b}}{N_{0}}\right)^{D, T}=\frac{W}{S R} \frac{k P^{D, T} r^{-v} / n_{\max }^{D}}{(1-\alpha) k P^{D, T} r^{-v}+\left(\sum_{j=1}^{c} I_{j}^{U, C}(r)+\sum_{k=1}^{b} I_{k}^{U, B}(r)+\sum_{l=1}^{L-(c+b)} I_{l}^{D, A}(r)\right)+P_{N}}
$$

In the numerical result, we will investigate the capacity and the size of inner zone of the target cell with different numbers of cells in each group.

\subsection{Capacity increase at target cell with adjacent cells in Group C}

It is clear that cells in Group C which don't reduce their service range generate higher intercell interference than cells in Group B. Therefore, the more cells in Group C are adjacent to a target cell, the less the capacity of the DL crossed slots in the target cell.

The high inter-cell interference from cells in Group C can be solved by employing an advanced time slot assignment in TDD. In a cell of Group C, by assigning UL slots which correspond to the DL crossed slots in a target cell only to those mobiles close to the BS, the 
interference can be reduced as in cells of Group B. UL slots that corresponds to UL in the target cell can be assigned to users away from the BS. By letting $S_{u}$ and $S_{c}$ be the number of nominal UL and DL crossed slots respectively, a cell in Group C can implement the advanced slot assignment with inner zone radius $r$ that satisfies $\pi r^{2} / \pi\left(D^{2}-r^{2}\right)=S_{c} /\left(S_{u}-S_{c}\right)$. Thus, we have

$$
r=D \sqrt{S_{c} / S_{u}}
$$

By assigning UL slots to mobiles within the range $r$ of cells in Group C, the target cell DL crossed slot capacity increases as in Equation (9), which is the case for cells in Group B.

\section{Numerical Results}

Numerical results of the analysis in Section 3 are presented with parameters in Table 2, which are widely accepted parameters in the mobile communication practice. $k$ and $v$ are selected to consider Okumura-Hata propagation model for urban area with BS height 30m, MS height 1.5m, and carrier frequency of $1950 \mathrm{MHz}$ [14]. Noise figure of 7dB for DL and $3 \mathrm{~dB}$ for $\mathrm{UL}$ is considered to calculate background noise power $P_{N}$. When considering thermal noise density of $-174 \mathrm{dBm}, \quad P_{N}=-174 \mathrm{dBm}+10 \log _{10}(W)+$ noise figure.

With the maximum transmission power of $10 \mathrm{~W}$ for BS and $200 \mathrm{~mW}$ for MS, we obtain $n_{\max }^{D}=5.16$ from $E_{b} / N_{0}$ value and load factor equation $[14,16]$ when every cell follows DL slot allocation. We also obtain $n_{\max }^{U}=4.93$. Thus, we assume that the target cell and cells in Group A serve $n_{\max }^{D}=5.16$ mobiles, and cells in Group B and C serve $n_{\max }^{U}=4.93$ mobiles at one time slot. We consider cell radius of $0.65 \mathrm{Km}$ throughout experiments since numerical results show similar trend for various cell radiuses.

Figure 4 shows the radius of inner zone of target cell that uses the downlink crossed slots. In the figure, 0B6C represents 0 cell in Group B and 6 cells in Group C consist of the adjacent 
Table 2. Parameters used in numerical experiments

\begin{tabular}{cc}
\hline Parameters & Values \\
\hline$W$ & $3840 \mathrm{Kbps}$ \\
$R$ & $8 \mathrm{Kbps}$ \\
$S$ & 15 \\
$L$ & 6 \\
$P_{N}$ & $-101.2 \mathrm{dBm}$ for DL \\
$v$ & $-105.2 \mathrm{dBm}$ for UL \\
$k$ & 3.5 \\
$\alpha$ & $-137.4 \mathrm{~dB}$ \\
$\gamma^{D}$ & 0.5 \\
$\gamma^{U}$ & $7 \mathrm{~dB}$ \\
\hline
\end{tabular}

cells. Note that cells in Group A are equivalent to those in Group C in terms of inter-cell interference. As the number of cells in Group B increases, the size of inner zone of the target cell increases. When all adjacent cells are in Group C, the size of inner zone becomes $58.8 \%$ of the cell radius. It is extended to $72.8 \%$ of cell radius if all six neighbors reduce their range by $50 \%$.

Figure 5 shows capacity increase in the target cell due to the reduced transmission range of neighbor cells in Group B. The capacity of target cell is 5.16 mobiles in a slot when all six neighbors do not reduce their transmission range. However, its capacity is increasing as the number of cells in Group B increases. Let us assume that the size of inner zone is $58.8 \%$ of the cell radius. Then, the number of mobiles reaches 7.56 when all six neighbor cells reduce their transmission range by $50 \%$.

Table 3 shows the capacity increase with the DL crossed slots. Compared to the nominal slot allocation which employs 8 DL timeslots and 7 UL, the DL capacity is increased by approximately $22 \mathrm{Kbps}$ for each crossed slot, when all six neighbor cells reduce their transmission range by $50 \%$. The table illustrates that the use of downlink crossed slots with 


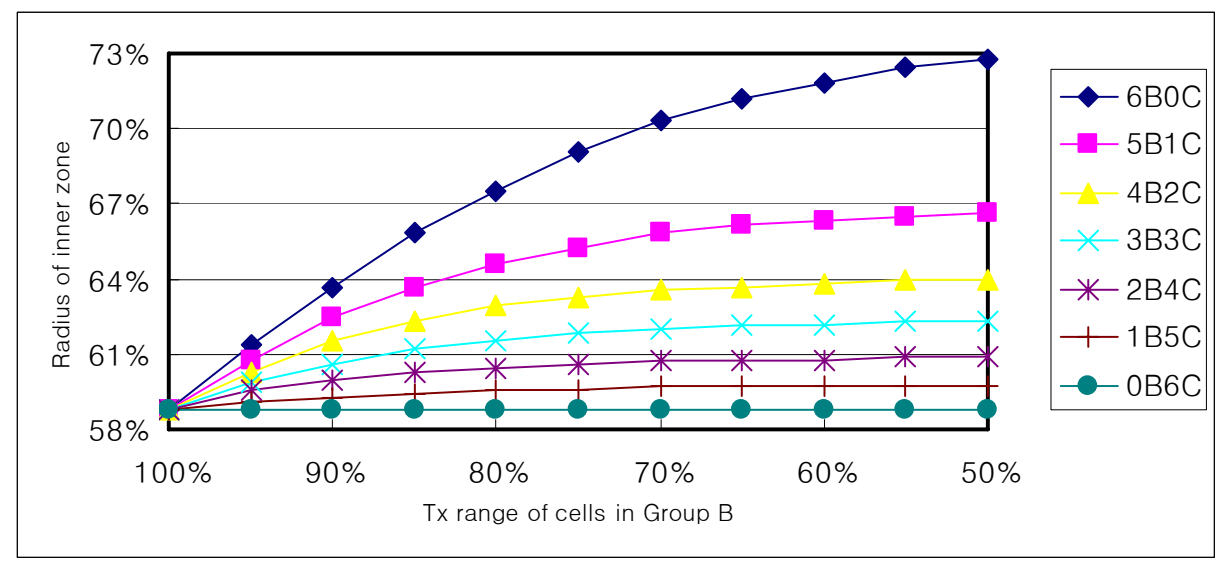

Figure 4. Radius of inner zone of target cell

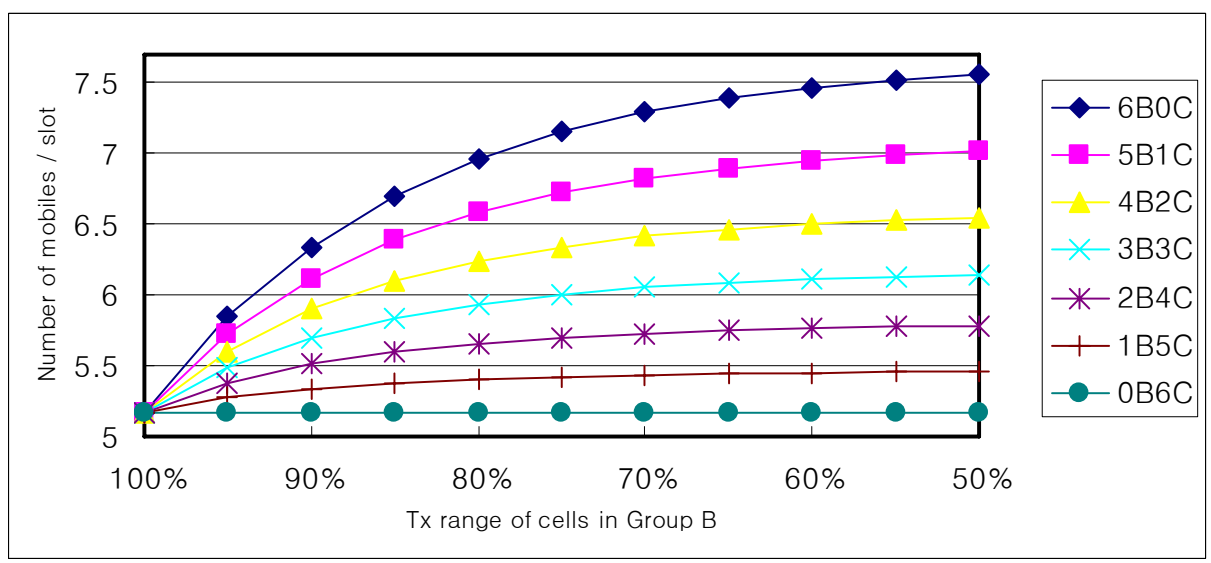

Figure 5. Number of mobiles in a downlink crossed slot

Table 3. Target cell transmission rate (Kbps) in a frame

\begin{tabular}{ccccccc}
\hline \multicolumn{3}{c}{ Number of time slots } & \multicolumn{2}{c}{ 0B6C } & \multicolumn{2}{c}{ 6B0C } \\
\hline $\begin{array}{c}\text { Regular } \\
\text { Uplink }\end{array}$ & $\begin{array}{c}\text { Regular } \\
\text { Downlink }\end{array}$ & $\begin{array}{c}\text { Crossed } \\
\text { Downlink }\end{array}$ & Uplink & Downlink & Uplink & Downlink \\
\hline 7 & 8 & 0 & 276.08 & 330.24 & 276.08 & 330.24 \\
\hline 6 & 8 & 1 & 236.64 & 344.51 & 236.64 & 352.12 \\
\hline 5 & 8 & 2 & 197.20 & 358.78 & 197.20 & 374.00 \\
\hline 4 & 8 & 3 & 157.76 & 373.06 & 157.76 & 395.87 \\
\hline
\end{tabular}


reduced transmission range in neighbor cells is a promising solution to tackle traffic asymmetry in a target cell.

Monte Carlo simulation is employed to verify numerical results. Parameters in Table 2 are used in the simulation. For the implementation of propagation loss, large scale fading is considered with the Okumura-Hata model. We assume that the large scale fading follows normal distribution in $\mathrm{dB}$ scale with mean of zero and standard deviation of $8.0 \mathrm{~dB}$.

Figure 6 shows the results of numerical analysis and simulation of two scenarios 3B3C and 6B0C. Similar results are obtained with other scenarios. From the figure it is clear that the radius of inner zone of target cell by the simulation well fits with the numerical analysis. The gap in the radius of inner zone of the two scenarios by the simulation is smaller than that by the analysis. This can be explained by the reduced interference effect by the cell nearest to the tagged mobile and the shadowing effect by other neighboring cells in Group C.

The effect of large scale fading is shown in Figure 7. It depicts the probability density function of inner zone radius, when the scenario 3B3C is employed with $70 \%$ of service range for cells in Group B. Although our simulation results well fit to the analysis, each cell has different radius of inner zone due to the different value of large scale fading. It implies that crossed slots can be more useful in cells with larger inner zone. In the figure, $14 \%$ of cells have inner zone radius of more than $0.8 D$, while the average inner zone radius is $0.62 D$.

\section{Conclusion}

The capacity of a DL crossed slot is investigated with various scenarios of adjacent cells. When all neighbor cells use nominal UL slots, the use of DL crossed slot in a target cell is restricted to an inner zone. The size of inner zone is determined by considering inter-cell interference and the minimum $E_{b} / N_{0}$ requirement. When the radius of the target cell is 0.65 $\mathrm{Km}$, the maximum size of inner zone $r$ that meets $\gamma^{P}=7 \mathrm{~dB}$ becomes $0.38 \mathrm{Km}$ which 


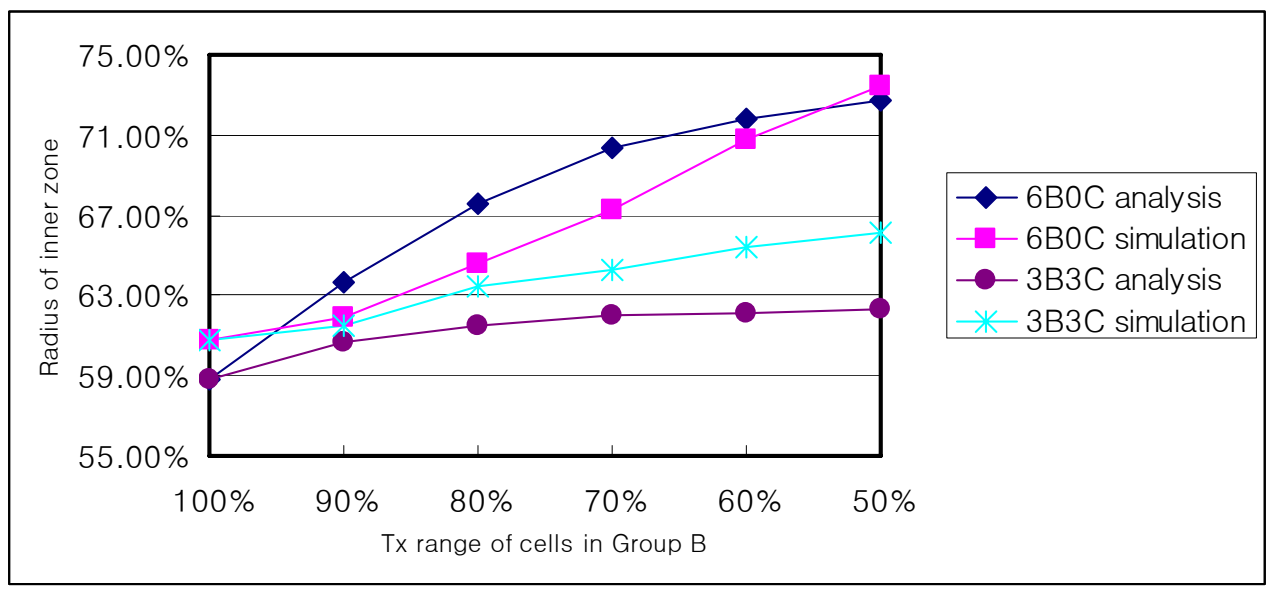

Figure 6. Comparison between analysis and simulation results

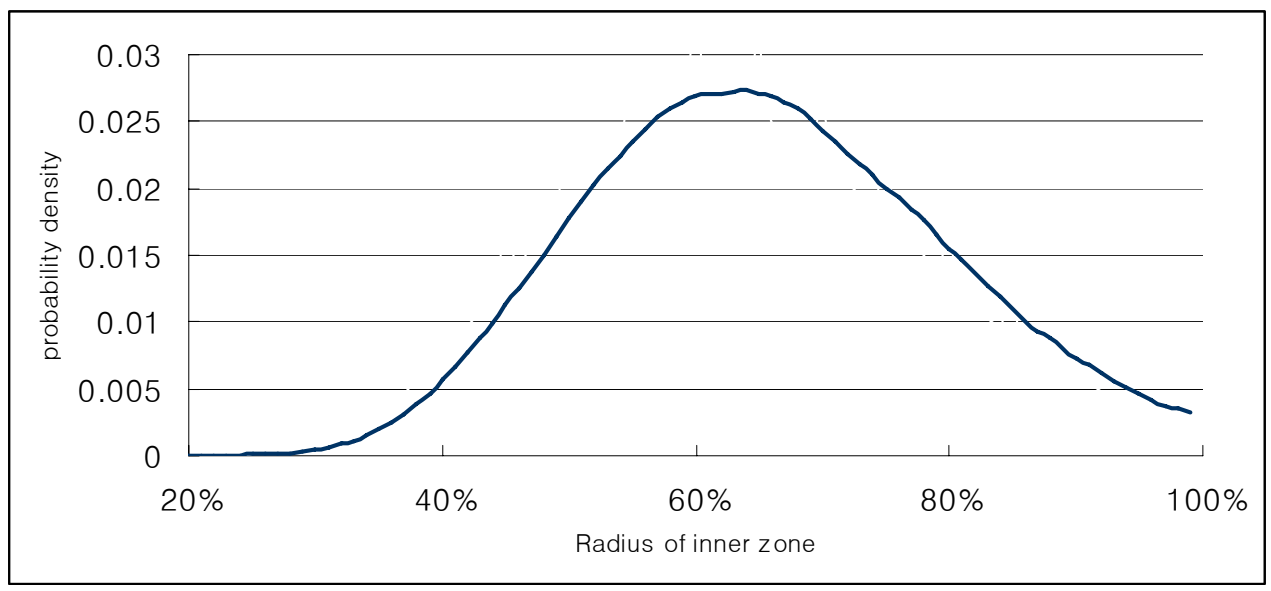

Figure 7. Probability density function of inner zone radius

corresponds to $58.8 \%$ of the cell radius.

The capacity increase in the target cell is examined by reducing the service range of neighbor cells. This situation, however, is possible when the traffic in neighbor cells is sparse and mobiles out of service range can be covered by UL timeslots that does not coincide with the crossed slots. When all six neighbors reduce their service range by $20 \%$, the capacity at target cell crossed slot is increased by $35 \%$. The increase is $46 \%$, when the service range at the neighbor cells is $50 \%$ of cell radius. Computational results also show that the increase of transmission rate by each additional DL crossed slot well compensates the decrease of UL transmission rate by reducing the service range in neighbor cells. 
Monte Carlo simulation is performed with large scale fading to verify the computational results. The results well supports our numerical analysis for the size of inner zone of a target cell that implements crossed slots.

\section{References}

[1] J. Nasreddine and X. Lagrange, Time Slot Allocation Based on a Path Gain Division Scheme for TD-CDMA TDD Systems, IEEE Vehicular Technology Conference (VTC), Spring, 2003.

[2] W. S. Jeon and D. G. Jeong, Comparison of Time Slot Allocation Strategies for CDMA/TDD Systems, IEEE Journal on Selected Areas in Communications, Vol. 18, No. 7, 2000.

[3] X. Wu, L. L. Yang, and L. Hanzo, Uplink Capacity Investigations of TDD/CDMA, IEEE Vehicular Technology Conference (VTC), Spring, 2002.

[4] L. C. Wang, S. Y. Huang, and Y. C. Tseng, Interference Analysis of TDD-CDMA Systems with Directional Antennas, IEEE Vehicular Technology Conference (VTC), pp. 1369-1373, Fall, 2003.

[5] Song Ni and Lajos Hanzo, Genetically Enhanced Performance of a UTRA-like TimeDivision Duplex CDMA Network, IEEE Vehicular Technology Conference, Spring, 2005

[6] J. S.Blogh and L. Hanzo, Third-Generation Systems and Intelligent Wireless Networking - Smart Antennas and Adaptive Modulation. John Wiley and IEEE Press, 2002.

[7] S. Ni, J. S. Blogh, and L. Hanzo, On the network performance of UTRA-like TDD and FDD CDMA systems using adaptive modulation and adaptive beamforming in Proceedings of the IEEE Vehicular Technology Conference, 2003, Spring, pp. 606-610, April, 2003.

[8] L. C. Godara, Application of antenna arrays to mobile communications, part 2: Beamforming and direction-of-arrival considerations, Proc. IEEE, Vol. 85, pp. 1195-1245, Aug. 
1997.

[9] F. Nazzarri and R. F. Ormondroyd, An Effective Dynamic Slot Allocation Strategy Based on Zone Division in WCDMA/TDD Systems, IEEE Vehicular Technology Conference, Fall, 2002

[10] J. Lee, Y. Han, and D. Kwon, An Adaptive Time Slot Allocation Strategy for WCDMA/TDD System, IEEE Vehicular Technology Conference, Spring, 2001.

[11] P. J. Czezowski, A. Morawej, and R. D McLeod, GPS Assisted Resource Allocation in Mobile Networks, Society for Computer Simulation, Vol.31 No.4, 1999

[12] H. C. Son, J. G. Lee, and G. I. Jee, Mobile Station Location Using Hybrid GPS and a Wireless Network, IEEE Vehicular Technology Conference, pp. 2716-2720, 2003

[13] M. Haardt, A. Klein, R. Koehn, S. Oestreich, M. Purat, V. Sommer, and T. Ulrich, The TD-CDMA Based UTRA TDD Mode, IEEE Journal on Selected Areas in Communications, Vol. 18, No. 8, 2000.

[14] H. Holma and A. Toskala, WCDMA for UMTS, $3^{\text {rd }}$ Edition, Wiley and Sons, 2004.

[15] D. Calin and M Areny, Impact of Radio Resource Allocation Policies on the TD-CDMA System Performance: Evaluation of Major Critical Parameters, IEEE Journal on Selected Areas in Communications, Vol. 19, No. 10, 2001.

[16] K. Sipilä, Z. C. Honkasalo, J. Laiho-Steffens, and A. Wacker, Estimation of Capacity and Required Transmission Power of WCDMA Downlink Based on a Downlink Pole Equation, IEEE Vehicular Technology Conference, 2000. 